\title{
LA INTERFERENCIA DE LA UTILIZACIÓN CONCURRENTE DE ALPRAZOLAM EN EL TRATAMIENTO COGNITIVO-COMPORTAMENTAL DEL TRASTORNO DE PÁNICO
}

\author{
AZUCENA GARCÍA-PALACIOS y CRISTINA BOTELLA \\ Universidad Jaime I
}

(Recibido el 6 de junio de 1997)

\begin{abstract}
El presente estudio analiza el papel de la utilización concurrente del alprazolam en la eficacia de la terapia cognitivo-comportamental para el trastorno de pánico. El trabajo se engloba dentro de un estudio previo más amplio. El objetivo de dicho estudio fue estudiar la eficacia diferencial de dos tratamientos, uno estándar y otro abreviado apoyado por manuales de autoayuda. Los resultados indicaron que ambos tratamientos eran igualmente eficaces. El objetivo del presente estudio es analizar la eficacia diferencial en función de si los pacientes tomaban o no alprazolam. Los resultados indican que, en prácticamente la totalidad de las variables analizadas, los sujetos mejoran por igual en el postest y en el seguimiento (a los doce meses de finalizada la terapia), independientemente de si empezaron el programa cognitivo-comportamental tomando o no un tratamiento farmacológico (alprazolam).
\end{abstract}

Palabras clave: Trastorno de pánico, terapia cognitivo-comportamental, alprazolam.

Interference of the use of alprazolam in the cognitive-behavioral treatment for panic disorder

This study analyzes the role of alprazolam in the therapeutic effectiveness of cognitive-behavioral treatments for panic disorder. This work belongs to a wider study which aim was to study the differential effectiveness of two programs, a standard one and a brief one supported by self-help manuals. We demonstrated the effectiveness of both interventions. The aim of the present study is to analyzed the differential effectiveness according to the use of alprazolam. The results shows that the patients who used alprazolam improve as well as patients that didn't use it in mostly of the analyzed variables at postest and follow-up assessment (12-month follow-up)

Key words: Panic disorder, cognitive-behavioral therapy, alprazolam.

\section{INTRODUCCIÓN}

Desde hace varios años se viene estudiando el diseño y puesta a prueba de tratamientos cognitivo-comportamentales para el trastorno de pánico. Estas alternativas de terapia han mostrado una eficacia superior a la condición de lista de espera, terapia de apoyo y placebo farmacológico (Clum, Clum y Surls, 1993,

\footnotetext{
Agradecimientos: Este trabajo se ha realizado gracias al proyecto número PB94-1093, financiado por la DGICYT (MEC). Queremos agradecer la inestimable colaboración de todos los miembros de la Unidad de Salud Mental de Vila-real, sin cuya ayuda no habría sido posible realizar esta investigación.
}

Gould, Otto y Pollack, 1995; Margraf, Barlow, Clark y Telch, 1993; Michelson y Marchione, 1991).

En la práctica clínica habitual resulta bastante común combinar el uso de tratamientos farmacológicos con tratamientos cognitivo-comportamentales para el trastorno de pánico. Lo usual es que, cuando un paciente acude a recibir un tratamiento psicológico para este trastorno, esté ya 
recibiendo fármacos. Los fármacos más utilizados han sido los antidepresivos tricíclicos, en concreto, la imipramina; los inhibidores de la mono-amino-oxidasa (IMAOs), como la fenelcina, y las benzodiacepinas, como el alprazolam. En los últimos años, también se han utilizado los inhibidores selectivos de la recaptación de serotonina (ISRSs), como la fluoxetina (McNally, 1996), y aunque hasta hace relativamente poco tiempo se consideraba a la imipramina como fármaco de elección (Barlow, 1988), últimamente algunos autores señalan que este lugar está siendo reemplazado por los ISRSs (p.ej., Klein, 1995).

Barlow (1988), después de hacer un análisis acerca de este tema, concluyó que los fármacos más útiles son los antidepresivos, concretamente la imipramina, y en especial si se quiere combinar con tratamientos psicológicos en los que se utilice la exposición, afirmación que comparten otros autores, como Marks y O'Sullivan (1992). Este autor también apela al "aprendizaje dependiente del estado", esto es: aquello que se ha aprendido bajo los efectos de una droga, no se generaliza a otros momentos o situaciones en los que ya no se está bajo esos efectos. Según esta afirmación, los fármacos causarían un efecto adverso en la consecución de los objetivos terapéuticos de un tratamiento psicológico. Esta idea, también denominada «la disociación farmacológica», está ampliamente extendida en Psicología clínica como una de las posibles limitaciones de la combinación de fármacos y tratamientos psicológicos (Echeburúa, 1997).

Por otro lado, también se ha afirmado que al combinar un tratamiento farmacológico con uno psicológico, el paciente puede atribuir la mejoría a los fármacos, lo que puede aumentar notablemente las probabilidades de que se produzca una recaída cuando finaliza el tratamiento. En este sentido, hace ya años, Davison y
Valins (1969) afirmaban que los pacientes que atribuyen el cambio terapéutico a los fármacos, no se sienten responsables de los cambios que se producen, consideran que el cambio no es "suyo" en un sentido psicológico y de esta forma, no es probable que mantengan los cambios una vez que concluye la medicación. En una línea similar, desde la perspectiva de la teoría de la autoeficacia de Bandura (1977), también se afirma que es más probable que los éxitos incrementen la autoeficacia si las ejecuciones se perciben como resultado de la habilidad más que como resultado de ayudas externas, en este caso, la medicación. Basoglu, Marks, Kilic, Brewin y Swinson (1994) realizaron un estudio empírico sobre la atribución de mejoría en un tratamiento combinado de alprazolam y exposición. Cuando los pacientes atribuían la mejoría a la medicación, este hecho se convertía en predictor de recaídas posteriores.

Existen algunos trabajos que tenían como objetivo el estudio de la eficacia de programas cognitivo-comportamentales para el trastorno de pánico, al encontrar que una parte de la muestra tomaba ya fármacos al iniciar el programa cognitivocomportamental, analizaron si este uso concurrente de medicación tenía alguna influencia en la eficacia del tratamiento psicológico (Coté Gauthier, Laberge, Cormier y Plamondon, 1994; Hecker, Losee, Fritzler y Fink, 1996; Oei, Llamas y Evans, 1997).

Coté et al., en un estudio sobre la eficacia diferencial de un tratamiento psicológico para el pánico frente a otro tratamiento de iguales características, pero con una reducción significativa del tiempo de contacto con el terapeuta, encontraron que, al comparar el grupo de pacientes que estaban tomando medicación (principalmente alprazolam) frente al grupo que no tomaba fármacos, no existían diferencias significativas en efi- 
cacia terapéutica en el postest, es decir, que la medicación no interfería en la consecución de los objetivos terapéuticos del tratamiento psicológico. Ambos grupos de sujetos mejoraban significativamente. Estos resultados se mantenían en el seguimiento a los 6 meses que estos autores realizaron.

Otro estudio más reciente (Hecker et al., 1996), en el que también se puso a prueba la eficacia diferencial de dos tratamientos cognitivo-comportamentales para el trastorno de pánico, analizó también el papel de la medicación en la eficacia terapéutica. En el pretest, los pacientes que tomaban medicación puntuaban de forma significativamente más elevada que los pacientes que no tomaban medicación en la medida global que ofrecía el cuestionario de miedos de Marks y Mathews (1979). Sin embargo, estas diferencias desaparecieron en el postratamiento y en el seguimiento. Además, tanto los pacientes que tomaban medicación como los que no lo hacian, obtenían mejorías significativas comparables en las demás medidas terapéuticas en el postest y en el seguimiento a los 6 meses.

Por último, Oei et al. (1997) realizaron un trabajo para analizar el efecto del uso de fármacos en un tratamiento cognitivocomportamental intensivo. Tampoco encontraron diferencias en la respuesta al tratamiento de los pacientes que recibieron sólo el programa cognitivo-comportamental y los que lo recibieron cuando ya estaban siguiendo un tratamiento farmacológico.

Por otra parte, existen otro tipo de estudios que han investigado empíricamente cuáles son los efectos de combinar ambos tratamientos como objetivo principal (Beurs, Balkom, Lange, Koele y van Dyke, 1995; Marks et al., 1993; Sharp et al., 1996). Beurs et al. (1995) y Sharp et al. (1996), utilizando como tratamiento farmacológico la fluvoxamina, concluyen que ambos tratamientos son eficaces y que la combinación de ambos parece ser más eficaz que los dos tratamientos por separado. Sin embargo, Marks et al. (1993) llevaron a cabo un estudio en el que analizaron la eficacia de un tratamiento combinado utilizando como fármaco el alprazolam. Estos autores encontraron que tanto el alprazolam como la exposición eran eficaces, pero, al acabar el tratamiento y en el seguimiento, los logros conseguidos con alprazolam se perdían y con la exposición se mantenían. Además, el tratamiento combinado no era más eficaz que los componentes por separado, sin embargo, los pacientes empeoraban al terminar el programa, al compararlos con los que recibieron exposición sin fármacos.

El trabajo que presentamos a continuación forma parte de un estudio más amplio cuyo objetivo era someter a prueba la eficacia diferencial de dos tratamientos cognitivo-comportamentales, un tratamiento de 10 sesiones de probada eficacia (Botella y Ballester, 1991) adaptado del programa cognitivo-comportamental de Clark y Salkovskis (1989) y de Barlow y Cerny (1988), y un programa de tratamiento abreviado (de 5 sesiones) apoyado por materiales de autoayuda, desarrollado por nosotros. Ambos tratamientos demostraron una eficacia similar (Botella y García-Palacios, en revisión; García-Palacios, 1997). Una vez comprobado lo anterior, nos planteamos la conveniencia de estudiar si existen diferencias en cuanto a eficacia entre los pacientes que no tomaban medicación y los que sí lo hacían, tomando la muestra completa de pacientes (tanto del tratamiento estándar como del abreviado). Es decir, nos interesa delimitar el papel que desempeña el uso concurrente de alprazolam en la eficacia terapéutica del tratamiento psicológico. 


\section{MÉTODO}

\section{Participantes}

La muestra utilizada para este estudio se recogió entre las personas que acuden al Servicio de Asistencia Psicológica de la Universidad Jaime I y a la Unidad de Salud Mental de Vila-real.

Se compuso de 23 pacientes ambulatorios. El rango de edad oscilaba entre 18 y 52 años, siendo la media de 29 años. Respecto al sexo, 18 eran mujeres $(78 \%)$ y 5 varones $(22 \%)$.

Para llevar a cabo el diagnóstico se utilizó la entrevista estructurada de Spitzer y Williams (1987) en la que aparecen los criterios del DSM-III-R para el trastorno por angustia. Todos los pacientes cumplían el diagnóstico de trastorno por angustia según el DSM-III-R (1987), 17 con agorafobia $(74 \%)$ y 6 sin agorafobia (26\%). En el momento en que se inició el estudio no se había publicado la $4^{\mathrm{a}}$ versión del DSM (DSM-IV, APA, 1994) Sin embargo, se realizó una valoración retrospectiva de los pacientes, una vez se dispuso del citado manual diagnóstico, y se encontró que, en ninguno de los casos, el diagnóstico variaba. 14 pacientes $(61 \%)$ estaban tomando medicación psicotrópica cuando se empezó el estudio. La medicación utilizada en todos los casos fue una benzodiacepina, en concreto alprazolam. Las dosis oscilaban desde $0.75 \mathrm{mg}$./día hasta $3 \mathrm{mg} . /$ día, siendo la media de consumo de $1.4 \mathrm{mg}$.

Se excluyó del estudio a las personas que no reunían los criterios de trastorno de pánico o que padecían alcoholismo, adicción a drogas, enfermedad orgánica severa, depresión o psicosis.

La media de la frecuencia semanal de ataques de pánico de la muestra total era de 2 ataques/semana, siendo el rango de 1 a 8 crisis semanales.

La media de la intensidad de las crisis era de 8 en una escala de 0 a 10, oscilando el rango de 6 a 10.
La duración media del trastorno era de 3 años y 5 meses y el rango oscilaba entre 1 mes y 14 años.

De los 23 pacientes que comenzaron el tratamiento abandonaron 3 (uno en la $2^{\mathrm{a}}$ sesión, otro en la $4^{\mathrm{a}}$ y el último en la $5^{\mathrm{a}}$ sesión).

\section{Procedimiento}

En este trabajo tomamos la muestra completa de pacientes que habían participado en el estudio previo (Botella y García-Palacios, en revisión; García-Palacios, 1997) y la dividimos en función del uso de alprazolam. Los que tomaban la medicación siguieron la instrucción dada en la evaluación antes de empezar la terapia de mantener la misma dosis y el mismo fármaco, o bien, si el paciente lo demandaba, de reducirla o de eliminarla, ajustándose a las directrices de su psiquiatra. Si alguno de los pacientes aumentaba la dosis o cambiaba a otro fármaco, era excluido del estudio.

Los grupos fueron los siguientes:

1. Grupo sin medicación $(\mathrm{N}=8)$ : Sujetos que no tomaron medicación en ningún momento del período que abarcó esta investigación.

2. Grupo con medicación $(\mathrm{N}=12)$ : Sujetos que, al inicio de la investigación ya seguían un tratamiento farmacológico.

Se establecieron tres momentos de evaluación, en los cuales se recogía información sobre las variables clínicas objeto de estudio. Estos momentos eran:

1. Antes del tratamiento.

2. Después del tratamiento.

3. Seguimiento a los doce meses de haber finalizado el tratamiento.

\section{Instrumentos utilizados}

Los instrumentos que utilizamos en nuestro estudio se exponen a continuación: 


\section{- Instrumentos semanales}

Diario de pánico (forma simple y ampliada): El paciente registra diariamente el número de ataques de pánico (tanto totales como de síntomas limitados), en qué situación se han dado, la duración, los síntomas que han aparecido y la intensidad de los ataques en una escala de 0 a 10. En la forma ampliada, se incluyen las interpretaciones negativas que se hacen de las principales sensaciones corporales que ha experimentado en cada ataque de pánico y el grado de creencia de las mismas, y la interpretación alternativa a las sensaciones corporales y en qué grado reevalúa la interpretación negativa.

Escala de Temor y Evitación Agorafóbicos (TEA), adaptada de Marks y Mathews (1979): Se establecen una serie de conductas objetivo que el paciente evita y se valora el grado de evitación en una escala de 0 a 10 , donde el 0 sería «Nunca lo evito" y el 10 "Siempre lo evito», y el grado de temor que le produce enfrentarse a cada una de las conductas objetivo en una escala de 0 a 10 , donde el 0 sería "Nada de miedo» y el 10 «Miedo extremon.

Medidas de mejoría clínica valoradas por el paciente y el terapeuta, adaptadas de Guy (1976):

Escalas de estado y mejoría del paciente valoradas por el terapeuta (EMT): Consta de dos escalas. En la primera de ellas (EMT1), el terapeuta valora, desde un punto de vista clínico, el estado global del paciente en una escala de 1 a 6 , desde «Normal», a «Muy gravemente perturbado". En la segunda escala (EMT2), el terapeuta valora la mejoría del paciente desde el comienzo del tratamiento en una escala de 1 a 7 desde "Mejoradísimo" a "Muchísimo peor».

Escala de mejoría valorada por el paciente (EMP): El paciente evalúa el grado de mejoría subjetiva que percibe des- de que comenzó el tratamiento en una escala igual a la EMT2, que va de 1 a 7 , donde 1 sería "Muchísimo mejor» y 7 «Muchísimo peor».

\section{- Medidas evaluadas en el pretest, postest y seguimientos}

Inventario de depresión de Beck (BDI) (Beck, Ward, Mendelson, Mock y Erbaugh, 1961), versión de 21 ítems, adaptado para la población española por Conde y Franch (1984).

Inventario de ansiedad Estado-Rasgo de Spielberger (STAI) (Spielberger, Gorsuch y Lushene, 1970), adaptado para la población española por TEA (1988).

Inventario de ansiedad de Beck (BAI) (Riskind, Beck, Brown y Steer, 1987), traducido por Ballester (1992). Este instrumento de 21 ítems mide el grado en que el paciente ha sufrido una serie de síntomas (21), durante la última semana. El paciente debe señalar la intensidad de cada uno de los síntomas de nada a mucho, pasando por poco y bastante.

Cuestionario de pensamientos distorsionados (CDI), traducido del cuestionario de Beck (Beck y Sokol-Kessler, 1986) por Ballester (1992). El paciente valora la presencia e intensidad en una escala de 4 puntos (nada, poco, bastante y mucho) de una serie de cogniciones relacionadas con el pánico en el momento de las crisis. El instrumento consta de 16 ítems.

Escala de adaptación (EAD) (Echeburúa y De Corral, 1987. En Borda y Echeburúa, 1991). El paciente evalúa en escalas de 0 a 5 (nada, casi nada, poco, bastante, mucho y muchísimo) el grado en que el problema interfiere en distintas áreas de vida (trabajo, vida social, tiempo libre, relación de pareja, y vida familiar). Por último, el paciente puntúa el grado de interferencia global del problema en su vida en una escala de las mismas características. 


\section{RESULTADOS}

En primer lugar se analizaron las posibles diferencias en las puntuaciones obtenidas en las variables estudiadas en la evaluación pretratamiento entre el grupo que tomaba medicación con el grupo que no tomaba medicación en la evaluación pretratamiento. Dada la probabilidad de que las medidas de ansiedad y depresión estuvieran altamente correlacionadas, se llevó a cabo un MANOVA, utilizando la Lambda de Wilks. Los resultados indicaron que no existían diferencias significativas entre los grupos en estas medidas (BDI, BAI,
STAI-E y STAI-R). Los contrastes indicaron que no se daban diferencias entre los grupos en ninguna de estas variables por separado. Para las otras variables se utilizaron pruebas paramétricas ( $t$ de Student) o no paramétricas ( $U$ de Mann-Whitney), según el tipo de variable a analizar. No se obtuvieron diferencias estadísticamente significativas entre los grupos en ninguna de las medidas estudiadas.

Las medias y desviaciones típicas de los dos grupos en cada variable a lo largo de los tres momentos de evaluación (pretest, postest y seguimiento) pueden observarse en la Tabla 1. Vemos que la

Tabla 1. Media y Desviación Típica de las variables en los grupos sin y con medicación (alprazolam) en los distintos momentos de evaluación

\begin{tabular}{|c|c|c|c|c|c|c|c|}
\hline \multirow[b]{2}{*}{ Variable } & \multirow[b]{2}{*}{ Grupos } & \multicolumn{2}{|c|}{ Pretest } & \multicolumn{2}{|c|}{ Postest } & \multicolumn{2}{|c|}{ Seguimiento } \\
\hline & & $M$ & $D T$ & $M$ & $D T$ & $M$ & $D T$ \\
\hline Crisis/mes & $\begin{array}{l}\text { sin med. } \\
\text { con med. }\end{array}$ & $\begin{array}{l}4,62 \\
5,25\end{array}$ & $\begin{array}{l}2,92 \\
2,56\end{array}$ & $\begin{array}{l}2,00 \\
0,58\end{array}$ & $\begin{array}{l}1,85 \\
1,00\end{array}$ & $\begin{array}{l}0,87 \\
0,16\end{array}$ & $\begin{array}{l}0,64 \\
0,39\end{array}$ \\
\hline Intensidad crisis & $\begin{array}{l}\text { sin med. } \\
\text { con med. }\end{array}$ & $\begin{array}{l}8,37 \\
7,50\end{array}$ & $\begin{array}{l}1,41 \\
1,38\end{array}$ & $\begin{array}{l}4,25 \\
2,00\end{array}$ & $\begin{array}{l}3,01 \\
3,16\end{array}$ & $\begin{array}{l}2,87 \\
0,67\end{array}$ & $\begin{array}{l}2,29 \\
1,30\end{array}$ \\
\hline № Síntomas & $\begin{array}{l}\text { sin med. } \\
\text { con med. }\end{array}$ & $\begin{array}{l}9,25 \\
7,75\end{array}$ & $\begin{array}{l}3,10 \\
2,67\end{array}$ & $\begin{array}{l}6,12 \\
1,75\end{array}$ & $\begin{array}{l}4,32 \\
3,33\end{array}$ & $\begin{array}{l}5,25 \\
1,58\end{array}$ & $\begin{array}{l}2,91 \\
1,56\end{array}$ \\
\hline Cogniciones (CDI) & $\begin{array}{l}\text { sin med. } \\
\text { con med. }\end{array}$ & $\begin{array}{l}29,50 \\
26,92\end{array}$ & $\begin{array}{l}3,70 \\
6,99\end{array}$ & $\begin{array}{l}18,62 \\
16,75\end{array}$ & $\begin{array}{l}3,20 \\
7,22\end{array}$ & $\begin{array}{l}15,00 \\
12,25\end{array}$ & $\begin{array}{l}4,57 \\
4,90\end{array}$ \\
\hline Evitación & $\begin{array}{l}\text { sin med. } \\
\text { con med. }\end{array}$ & $\begin{array}{l}8,50 \\
8,10\end{array}$ & $\begin{array}{l}1,38 \\
1,76\end{array}$ & $\begin{array}{l}2,67 \\
2,33\end{array}$ & $\begin{array}{l}2,50 \\
2,55\end{array}$ & $\begin{array}{l}2,83 \\
1,67\end{array}$ & $\begin{array}{l}2,64 \\
2,60\end{array}$ \\
\hline Temor & $\begin{array}{l}\text { sin med. } \\
\text { con med. }\end{array}$ & $\begin{array}{l}6,67 \\
7,22\end{array}$ & $\begin{array}{l}1,37 \\
2,17\end{array}$ & $\begin{array}{l}3,17 \\
2,33\end{array}$ & $\begin{array}{l}2,23 \\
1,50\end{array}$ & $\begin{array}{l}2,83 \\
1,67\end{array}$ & $\begin{array}{l}2,99 \\
1,12\end{array}$ \\
\hline STAI-E & $\begin{array}{l}\text { sin med. } \\
\text { con med. }\end{array}$ & $\begin{array}{l}28,75 \\
22,42\end{array}$ & $\begin{array}{l}10,65 \\
11,32\end{array}$ & $\begin{array}{l}19,37 \\
15,75\end{array}$ & $\begin{array}{l}8,28 \\
5,59\end{array}$ & $\begin{array}{l}20,37 \\
17,25\end{array}$ & $\begin{array}{r}8,28 \\
11,07\end{array}$ \\
\hline STAI-R & $\begin{array}{l}\text { sin med. } \\
\text { con med. }\end{array}$ & $\begin{array}{l}37,50 \\
32,08\end{array}$ & $\begin{array}{l}6,70 \\
8,15\end{array}$ & $\begin{array}{l}30,12 \\
22,67\end{array}$ & $\begin{array}{l}6,57 \\
6,05\end{array}$ & $\begin{array}{l}30,87 \\
24,25\end{array}$ & $\begin{array}{l}5,94 \\
9,35\end{array}$ \\
\hline $\mathrm{BAI}$ & $\begin{array}{l}\text { sin med. } \\
\text { con med. }\end{array}$ & $\begin{array}{l}24,37 \\
22,83\end{array}$ & $\begin{array}{l}7,73 \\
9,01\end{array}$ & $\begin{array}{r}21,12 \\
8,75\end{array}$ & $\begin{array}{l}6,81 \\
4,97\end{array}$ & $\begin{array}{l}17,00 \\
10,25\end{array}$ & $\begin{array}{l}7,27 \\
4,67\end{array}$ \\
\hline BDI & $\begin{array}{l}\text { sin med. } \\
\text { con med. }\end{array}$ & $\begin{array}{l}16,37 \\
15,64\end{array}$ & $\begin{array}{l}6,95 \\
9,38\end{array}$ & $\begin{array}{r}11,62 \\
6,36\end{array}$ & $\begin{array}{l}5,75 \\
3,50\end{array}$ & $\begin{array}{l}13,12 \\
10,27\end{array}$ & $\begin{array}{l}4,12 \\
6,84\end{array}$ \\
\hline Estado del paciente & $\begin{array}{l}\text { sin med. } \\
\text { con med. }\end{array}$ & $\begin{array}{l}3,25 \\
3,42\end{array}$ & $\begin{array}{l}0,70 \\
0,67\end{array}$ & $\begin{array}{l}2,25 \\
2,08\end{array}$ & $\begin{array}{l}0,46 \\
0,51\end{array}$ & $\begin{array}{l}1,87 \\
1,67\end{array}$ & $\begin{array}{l}0,64 \\
0,65\end{array}$ \\
\hline Mejoría terapeuta & $\begin{array}{l}\text { sin med. } \\
\text { con med. }\end{array}$ & $\begin{array}{l}4,25 \\
4,08\end{array}$ & $\begin{array}{l}0,46 \\
0,29\end{array}$ & $\begin{array}{l}2,12 \\
2,25\end{array}$ & $\begin{array}{l}0,35 \\
0,45\end{array}$ & $\begin{array}{l}2,00 \\
1,58\end{array}$ & $\begin{array}{l}0,53 \\
0,67\end{array}$ \\
\hline Mejoría paciente & $\begin{array}{l}\text { sin med. } \\
\text { con med. }\end{array}$ & $\begin{array}{l}3,87 \\
3,92\end{array}$ & $\begin{array}{l}0,35 \\
0,29\end{array}$ & $\begin{array}{l}1,62 \\
1,33\end{array}$ & $\begin{array}{l}0,52 \\
0,49\end{array}$ & $\begin{array}{l}1,37 \\
1,25\end{array}$ & $\begin{array}{l}0,74 \\
0,45\end{array}$ \\
\hline Interferencia global & $\begin{array}{l}\text { sin med. } \\
\text { con med. }\end{array}$ & $\begin{array}{l}3,12 \\
3,50 \\
\end{array}$ & $\begin{array}{l}0,83 \\
0,67 \\
\end{array}$ & $\begin{array}{l}2,5 \\
1,67\end{array}$ & $\begin{array}{l}0,75 \\
0,65 \\
\end{array}$ & $\begin{array}{l}1,37 \\
1,25 \\
\end{array}$ & $\begin{array}{l}0,74 \\
0,62 \\
\end{array}$ \\
\hline
\end{tabular}

Nota: STAI-E = Inventario de ansiedad-estado de Spielberger. STAI-R = Inventario de ansiedad-rasgo de Spielberger. $\mathrm{BAI}=$ Inventario de ansiedad de Beck. BDI = Inventario de depresión de Beck. 
evolución en los dos grupos (sin medicación y con medicación) sigue el mismo patrón, se produce un descenso significativo de las puntuaciones medias en el postest y este cambio se mantiene en el seguimiento (ver Figuras 1 y 2 , en el que se ofrece la evolución de los sujetos en dos variables: Mejoría percibida por el paciente y Mejoría percibida por el terapeuta).

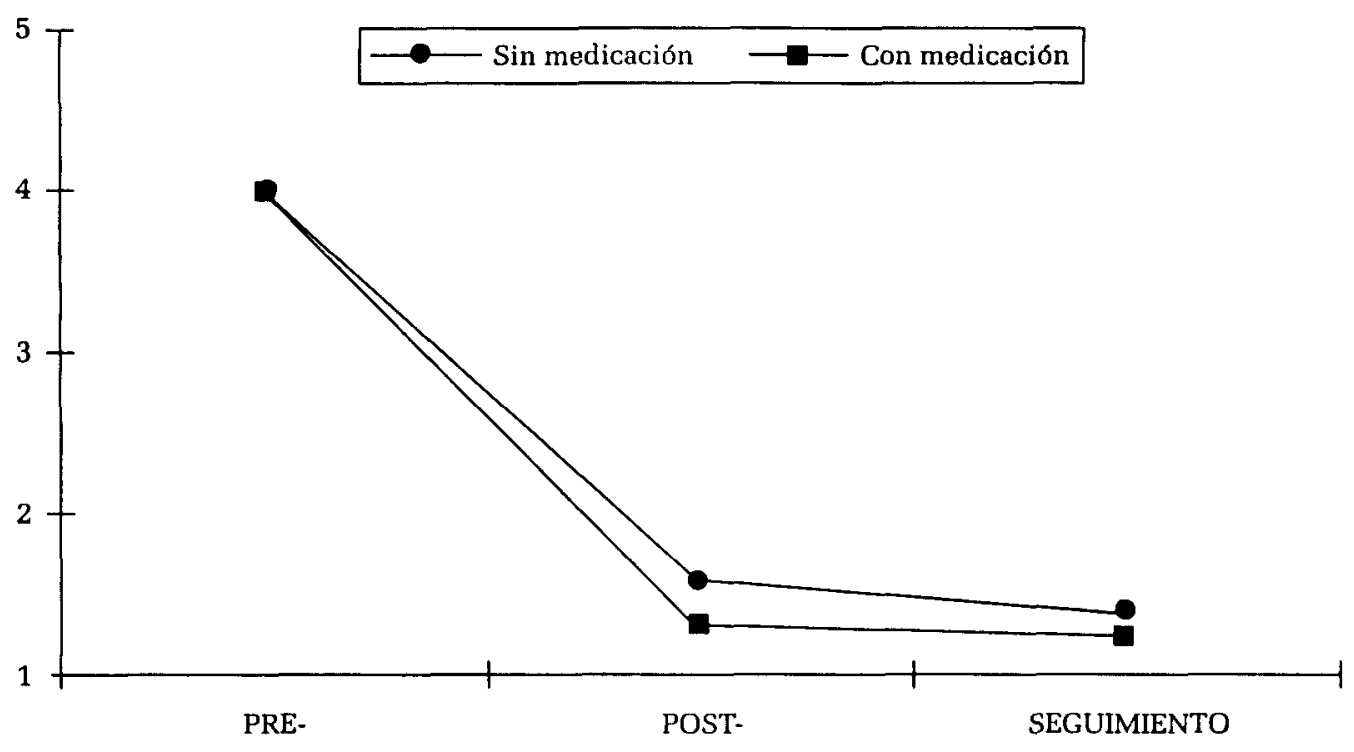

Figura 1. Evolución de los grupos a lo largo del proceso terapéutico en la medida de mejoría valorada por el paciente

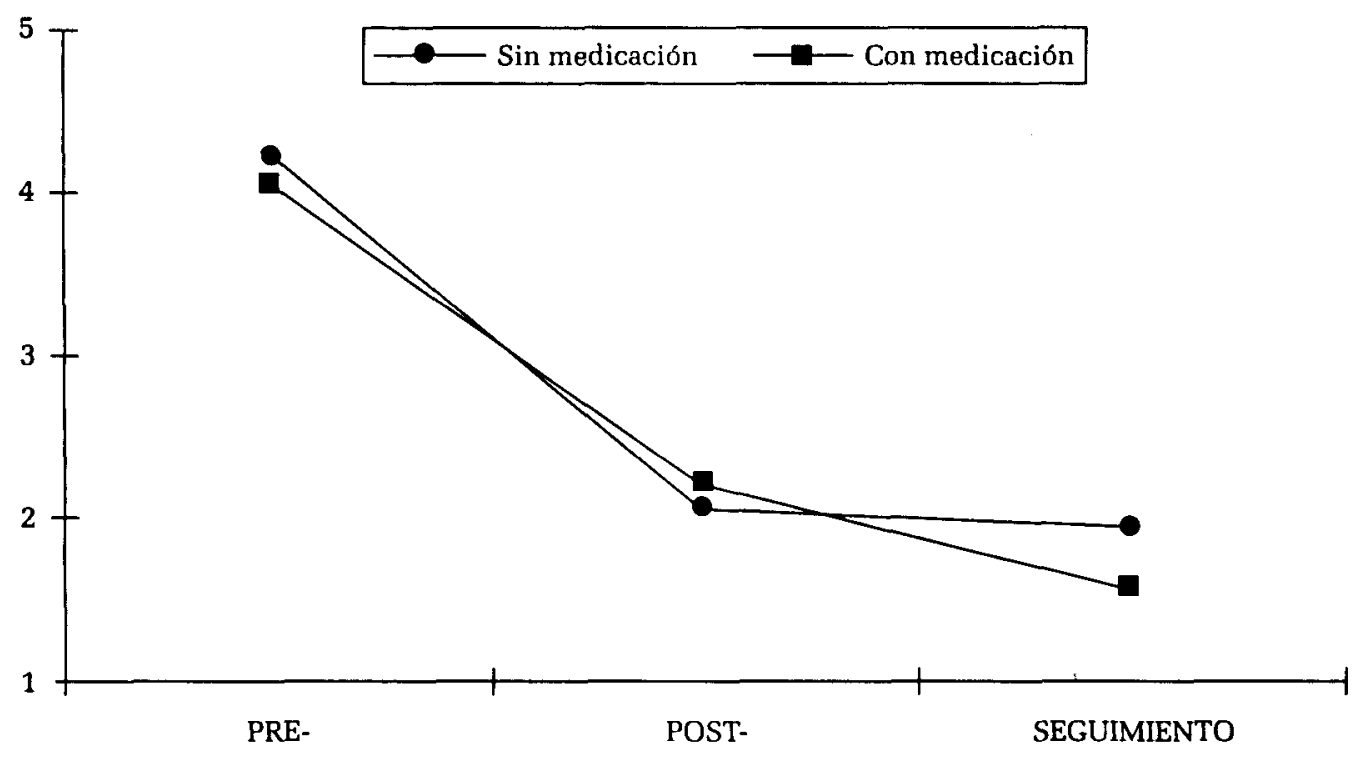

Figura 2. Evolución de los grupos a lo largo del proceso terapéutico en la medida de mejoría del paciente valorada por el terapeuta 
Se realizaron distintos análisis (paramétricos y no paramétricos) para analizar las posibles diferencias en la eficacia terapéutica según si existía un uso concurrente de medicación o no. Veamos los resultados:

Medidas de estatus clínico: Agrupamos algunas variables que consideramos muy relevantes para valorar el estatus clínico del paciente, tres medidas objetivas: el número de crisis al mes, variable considerada central como medida de cambio en el trastorno de pánico, el número de síntomas, muy relacionada con la anterior, y las cogniciones catastróficas medidas por el CDI, variable con gran importancia teórica desde una perspectiva cognitiva del trastorno de pánico. Incluimos también una medida subjetiva, ya que entendíamos era importante para la valoración del estatus clínico del paciente, el estado del paciente valorado por el terapeuta. Se llevó a cabo un MANOVA de medidas repetidas, utilizando la lambda de Wilks. No se encontraron efectos significativos para el grupo (Tamaño efecto (TE) $=0,471$; potencia $(P)=0,706)$. Sin embargo, los contrastes univariados indicaron que el efecto grupo (medicación) resultó significativo en una de las variables, número de sintomas, $\quad F(1,18)=12,51, \quad p<0,002$, ( $\mathrm{TE}=0,410 ; \mathrm{P}=0,917)$. En esta medida las puntuaciones eran más bajas en el grupo con uso concurrente de medicación, durante todo el proceso terapéutico. Respecto al efecto tiempo, éste fue significativo, $F(8,11)=24,08, p<0,000$, (TE=0,946; $\mathrm{P}=1,00$ ). Los contrastes revelaron diferencias significativas entre el pretest y el postest en todas las variables y el cambio se mantenía en el seguimiento. Por último, no se produjo un efecto interacción Grupo $x$ Tiempo estadísticamente significativo ( $\mathrm{TE}=0,438 ; \mathrm{P}=0,286$ ). Como vemos, en estas variables el uso concurrente de medicación no afectó a la respuesta al tratamiento psicológico de los pacientes.
Medidas de ansiedad y depresión: También se realizó un MANOVA para estas variables. No se produjo efecto Grupo $(\mathrm{TE}=0,477 ; \mathrm{P}=0,717)$. Sí existe un efecto significativo para el tiempo, $F(8,11)=$ 9,22, $p<0,001$, (TE=0,870; $\mathrm{P}=0,998$ ). Los contrastes indicaron que se producía un cambio significativo en el postest, que se mantenía en el seguimiento en todas estas variables. Por último, el MANOVA tampoco encuentra un efecto interacción significativo ( $\mathrm{TE}=0,568 ; \mathrm{P}=0,477$ ). Estos resultados indican que no aparecen diferencias entre los grupos en estas variables de ansiedad general, y no aparece influencia de la medicación en la eficacia terapéutica.

El resto de las medidas se analizaron mediante pruebas no paramétricas. Se utilizó la prueba de Friedman para estudiar los cambios desde el pretest al seguimiento. Utilizamos la prueba de los rangos con signo de Wilcoxon para las diferencias entre los distintas evaluaciones. Finalmente, se realizaron $U$ de MannWhitney para analizar las diferencias entre las condiciones experimentales.

Medidas directamente relacionadas con el pánico: Respecto a la intensidad de las crisis, la prueba de Friedman reveló diferencias estadísticamente significativas $(\chi(2, n=20)=34,51, p=0,000)$. Los análisis de Wilcoxon señalaron diferencias entre el pretest y el postest, $(z=-3,93$, $p=0,000$ ), pero no existían diferencias entre el postest y el seguimiento. Con respecto a las diferencias entre los grupos, las pruebas $U$ de Mann-Whitney no señalaron diferencias significativas, ni en el pretest ni en el postratamiento, aunque sí en el seguimiento, $(z=-2,37, p=0,031)$. En esta última evaluación, la intensidad de las crisis era valorada como significativamente menor en el grupo con uso concurrente de medicación. Los análisis realizados con la evitación agorafóbica revelaron diferencias desde el pretest al 
seguimiento $(\chi(2, n=15)=24,10$, $p=0,000$ ). La prueba de Wilcoxon halló diferencias entre el pretest y el postest, $(z=-3,41, p=0,001)$, y no las encontró entre el postest y el seguimiento. Los análisis $U$ de Mann-Whitney no encontraron diferencias entre las dos condiciones experimentales en ninguna de las evaluaciones. Los resultados respecto al temor agorafóbico fueron muy similares. En primer lugar, se produjo un cambio significativo desde el pretest al seguimiento, $(\chi(2, n=15)=24,94, p=0,000)$. La reducción significativa en esta variable se produjo desde el pretest al postest $(z=$ $-3,42, p=0,001)$ y se mantuvo en el seguimiento. Por otro lado, no se produjeron diferencias significativas entre los grupos en ningún momento del proceso.

Medidas relacionadas con la mejoría y la interferencia del problema en la vida del paciente: Los análisis revelan un cambio significativo desde el pretest al seguimiento en la mejoría del paciente valorada por el terapeuta, $(\chi(2, n=20)=$ $36,11, p=0,000)$. Existen diferencias significativas entre el pretest y el postest, $(z=$ $-4,05, p=0,000$ ) y se observa que los pacientes siguen mejorando por las diferencias encontradas entre el postest y el seguimiento $(z=-2,49, p=0,013)$. Las pruebas $U$ de Mann-Whitney señalan que no se observan diferencias entre los grupos. En cuanto a la mejoría valorada por el propio paciente, encontramos diferencias desde el pretest al seguimiento, $(\chi(2, n=20)=35,04, p=0,000)$. Estas diferencias se observan entre el pre y el postratamiento, $(z=-4,04, p=0,000), y$ ya no se producen entre el postest y el seguimiento. Por otro lado, no se producen diferencias entre las condiciones experimentales. Por último, también se observa un cambio significativo desde el pretest al seguimiento en la interferencia global, $(\chi(2, n=20)=32,09, p=0,000)$. Los análisis revelaron diferencias entre el pre y el postest $(z=-3,58, p=0,000)$ y también entre el postest y el seguimiento, $(z=-2,49, p=0,013)$, siendo menores las puntuaciones en el seguimiento. Por último, respecto a las diferencias entre los grupos en la interferencia global, éstas no se produjeron en el pretest, aunque sí en el postratamiento $(z=-2,29, p=0,031)$, siendo menores las puntuaciones en el grupo con alprazolam. Sin embargo, estas diferencias se diluyen en el seguimiento.

\section{DISCUSIÓN}

En primer lugar, consideramos importante señalar el hecho de que en la evaluación pretest no aparezcan diferencias significativas en ninguna de las variables entre los pacientes que tomaban medicación y los que no lo hacían antes de aplicar los tratamientos.

En segundo lugar, los análisis nos dicen, como ya comprobamos en un estudio anterior (García-Palacios, 1997), que se ha producido un cambio significativo en todas las variables comparando el pretest con el postest, y ese cambio se mantiene en los seguimientos, por lo que podemos concluir que los tratamientos utilizados han sido eficaces a la hora de tratar el trastorno de pánico, lo que va en la línea de las investigaciones que afirman que los tratamientos cognitivo-comportamentales son eficaces para tratar este trastorno (Acierno, Hersen y van Hasselt, 1993; Clum, 1989; Clum et al., 1993; Gould et al., 1995; Michelson y Marchione, 1991).

Los análisis nos indican que los pacientes que tomaban medicación obtenían puntuaciones significativamente menores en una de las variables, el número de síntomas, durante todo el proceso terapéutico. Una explicación plausible sería que la ingesta de medicación hace disminuir la intensidad y los síntomas específicos de los ataques de 
pánico de estos pacientes. Algo, por otra parte, totalmente esperable.

Por lo que respecta al objetivo fundamental del presente trabajo, los resultados nos ofrecen evidencia de que no existen diferencias estadísticamente significativas en la respuesta al tratamiento entre el grupo que tomaba alprazolam concurrentemente con el tratamiento cognitivo-comportamental y el que no lo hacía en casi todas las variables objeto de estudio, que engloban, tanto variables clínicas generales de ansiedad y depresión, como variables más directamente relacionadas con el estatus clínico del paciente y el trastorno en sí: la frecuencia, número de síntomas, cogniciones propias del pánico, el estado global del paciente, la intensidad de las crisis y la evitación y el temor agorafóbicos; y, por último, como variables de mejoría valorada tanto por el paciente como por el terapeuta y de interferencia. Las únicas medidas en que sí se encuentran diferencias entre los sujetos que tomaban medicación y los que no, son: la intensidad de las crisis y el nivel de interferencia global. En ambos casos encontramos una mayor reducción después del tratamiento en el grupo que tomaba alprazolam.

Por lo que respecta al nivel de interferencia global, parece que ésta es menor en el grupo que usaba fármacos después de finalizada la terapia. Sin embargo, al igual que en el BAI, estas diferencias desaparecen en los seguimientos.

Por último, en la intensidad de las crisis no aparecen diferencias hasta el seguimiento. En ese momento los pacientes que utilizaban alprazolam valoran sus crisis como menos intensas. Es posible que el efecto de la medicación haya influido en que los pacientes experimenten sus ataques de pánico como menos intensos.

También nos gustaría señalar el hecho de que no se observe influencia en la eficacia terapéutica del uso concurrente de alprazolam en otras variables muy relevantes en este campo, como son la frecuencia y el número de síntomas, el nivel de depresión y la mejoría valorada por el paciente y por el terapeuta.

En general, podemos concluir, que no existen diferencias importantes en la respuesta al tratamiento entre los pacientes que tomaron de forma concurrente medicación (alprazolam) o no la tomaron al seguir un tratamiento cognitivo-comportamental para el pánico. El alprazolam no perturbó la eficacia del programa cognitivo-comportamental, y si tuvo algún efecto en el mismo éste fue beneficioso para los pacientes. Estos datos van en contra de la idea de Barlow (1988) y de los resultados de Marks et al. (1993) que señalan que el uso de medicación, en concreto benzodiacepinas, puede interferir negativamente en los resultados de la terapia cognitivo-comportamental, y apoyan los resultados de Côté et al. (1994), Hecker et al. (1996) y Oei et al. (1997) que tampoco encuentran diferencias.

Es decir, nuestros datos van en contra de la idea del aprendizaje dependiente del estado o de la disociación farmacológica y de las atribuciones del paciente respecto a la eficacia terapéutica. Respecto a este último punto también queremos añadir que, aunque resulte sugerente desde una perspectiva psicológica afirmar que el uso concurrente de medicación pueda perturbar una atribución adecuada, nuestros datos no lo apoyan. Quizás la explicación esté en las instrucciones que se les daban a los pacientes durante todo el proceso de terapia y, más detalladamente, en el módulo de prevención de recaídas sobre el papel activo que desempeñaban en su propio tratamiento. Creemos que este énfasis ha podido ayudar a que los pacientes atribuyeran la mejoría a su trabajo y su esfuerzo en la práctica de las técnicas cognitivo-comportamentales, más que a otros elementos externos como la medicación. Por último, nos gustaría 
señalar que nosotros hemos obtenido estos datos en un estudio en que el tratamiento de base era el psicológico y se aconsejaba a los pacientes que fueran disminuyendo el tratamiento farmacológico, en la medida en que lo consideraran posible y de acuerdo con su psiquiatra. Es decir, no se trata de un tratamiento que desde el principio se haya pautado combinando fármacos y terapia cognitivocomportamental, sino de la aplicación de un programa psicológico a pacientes que podían o no estar tomando medicación. Lo mismo ocurre en los estudios de Côté et al., (1994), Hecker et al. (1996) y Oei et al., (1997). Existen otros estudios que sí estudian la influencia de benzodiacepinas, por ejemplo el de Marks et al. (1993), que encontró que el alprazolam sí interfería en el mantenimiento de los logros conseguidos con exposición. Sin embargo, se deberían realizar más estudios en esta línea que analizaran el efecto del alprazolam en tratamientos que combinaran un tratamiento farmacológico y un programa psicológico, sobre todo tratamientos cognitivo-comportamentales, siendo esta combinación la base de la terapia, para poder ser más concluyentes en el papel que desempeña la utilización de este fármaco en los tratamientos psicológicos.

\section{REFERENCIAS BIBLIOGRÁFICAS}

American Psychiatric Association (1987).

Diagnostical and Statistical Manual of Mental Disorders ( $3^{\mathrm{a}}$ ed. rev.). Washington, DC: APA (Barcelona, Masson, 1988).

American Psychiatric Association (1994). Diagnostical and Statistical Manual of Mental Disorders ( $4^{\mathrm{a}}$ ed.), Washington, DC: APA (Barcelona, Masson, 1995).

Ballester, R. (1992). Análisis de los actuales planteamientos cognitivos acerca del trastorno de pánico y el concepto de conducta de enfermedad. Tesis Doctoral. Universidad de Valencia.
Bandura, A. (1977). Social learning theory. Englewood Cliffs, N.J.: Prentice-Hall.

Barlow, D.H. (1988). Anxiety and its disorders: The nature and treatment of anxiety and panic. New York: Guilford Press.

Basoglu, M., Marks, I.M., Cengiz, K., Brewin, C.R. y Swinson, R.P. (1994). Alprazolam and exposure for panic disorder with agoraphobia. Attribution of Improvement to medication predicts subsequent relapse. British Journal of Psychiatry, 164, 652-659.

Barlow, D.H. y Cerny, J.A. (1988): Psychological Treatment of Panic. New York: Guilford Press.

Beck, A.T. y Sokol-Kessler, L. (1986). A test of cognitive dysfunction in panic attacks. Paper presented at the research conference, University of Pennsylvania, Philadelphia.

Beck, A.T., Ward, C.H., Mendelsohn, M., Mock, J. y Erbaugh, J. (1961). An inventory for measuring depression. Archives of General Psychiatry, 4, 561-571.

Beurs, E., Balkom, A., Lange, A., Koele, P. y van Dyke, R. (1995). Treatment of panic disorder with agoraphobia: Comparisons of fluvoxamine, placebo, and psychological panic management combined with exposure and of exposure in vivo alone. American Journal of Psychiatry, 15, 683-691.

Borda, M. y Echeburúa, E. (1991). La autoexposición como tratamiento psicológico en un caso de agorafobia. Análisis y Modificación de Conducta, 18, 101-103.

Botella, C. y Ballester, R. (1991). Tratamiento psicológico del trastorno de pánico: adaptación del programa cognitivo-conductual de Clark. Análisis y Modificación de Conducta, 17, 871-894.

Botella, C. y García-Palacios, A. (en revisión). Brief and Reduced Therapist Contact Program for the Treatment of Panic: Effectiveness in the treatment of panic disorder. Behavioural and cognitive Psychotherapy.

Clark, D.M. y Salkovskis, P.M. (1989). Cognitive therapy for panic and hypocondriasis. Oxford: Pergamon.

Clum, G.A. (1989). Psychological interventions vs. drugs in the treatment of panic. Behavior Therapy, 20, 429-457.

Clum, G.A., Clum, G.A. y Surls, R. (1993). A meta-analysis of treatments for panic disor- 
der. Journal of consulting and Clinical Psychology, 61, 317-326.

Conde, V. y Franch, J.I. (1984). Escalas de evaluación comportamental para la cuantificación de la sintomatología psicopatológica en los trastornos angustiosos y depresivos. Upjohn.

Côté, G., Gauthier, J.G., Laberge, B., Cormier, H.J. y Plamondon, J. (1994). Reduced therapist contact in the cognitive behavioral treatment of panic disorder. Behavior Therapy, 25, 123-145.

Davison, G. y Valins, S. (1969). Maintenance of self-attributed and drug-attributed behavior change. Journal of Personality and Social Psychology, 11, 25-33.

Echeburúa, E. (1997) Terapia de conducta y psicofarmacología: nuevas vías de integración. $5^{2}$ Congreso Nacional de Psicología Clínica y de la Salud. Centro Clínico Los Naranjos. Banalmádena.

García Palacios, A. (1997). Eficacia diferencial de un programa de tratamiento abreviado para el trastorno de pánico. Tesis Doctoral. Universidad Jaime I.

Gould, R.A., Otto, M.W. y Pollack, M.H. (1995). A meta-analysis of treatment outcome for panic disorder. Clinical Psychology Review, 15, 819-844.

Guy, W. (1976). ECDEU. Assessment Manual for Psychopharmacology Revised. NIMH Publ. DHEW Publ. No (Adm) 76-338.

Hecker, J.E., Losee, M.C., Fritzler, B.K. y Fink, C.M. (1996). Self-directed versus therapistdirected cognitive behavioral treatment for panic disorder. Journal of Anxiety Disorders, 10, 253-265.

Klein, D.F. (1995). Comunicación personal, 5 de septiembre.

Margraf, J., Barlow, D.H., Clark, D.M. y Telch, M.J. (1993). Psychological treatment of panic: work in progress on outcome, active ingredients, and follow-up. Behaviour Research and Therapy, 31, 1-8.

Marks, I.M. y Mathews, A.M. (1979). Brief standard rating for phobic patients. Behaviour Research and Therapy, 24, 83-85.

Marks, I.M. y O'Sullivan, G. (1992). Psicofármacos y tratamientos psicológicos en la agorafobia/pánico y en los trastornos obsesivo-compulsivos. En E. Echeburúa (Ed.) Avances en el tratamiento psicológico de los trastornos de ansiedad. Madrid: Pirámide.

Marks, I.M., Grey, S., Cohen, S.D., Hill, R., Mawson, D., Ramm, E.M. y Stern, R.S. (1983). Imipramine and brief therapistaided exposure in agoraphobics having self exposure homework: A controlled trial. Archives of General Psychiatry, 40, 153162.

Marks, I.M., Swinson, R.P., Basoglu, M., Kuch, K., Noshirvani, H., O'Sullivan, G., Lelliot, P.T., Kirby, M., McNamee, G., Sengun, S. y Wickwire, K. (1993). Alprazolam and exposure alone and combined in panic disorder with agoraphobia. A controlled study in London and Toronto. British Journal of Psychiatry, 162, 776-787.

McNally, R.J. (1996). Nuevos desarrollos en el tratamiento del trastorno de pánico. Revista de Psicopatología y Psicología Clínica, 1, 91-103.

Michelson, L.K. y Marchione, K. (1991). Behavioral, cognitive, and pharmacological treatments of panic disorder with agoraphobia: Critique and synthesis. Journal of Consulting and Clinical Psychology, 59, 100-114.

Oei, T.P.S., Llamas, M. y Evans, L. (1997). Does concurrent drug intake affect the long-term outcome of group cognitive behaviour therapy in panic disorder with or without agoraphobia? Behaviour Research and Therapy, 35, 851-857.

Riskind, J.H., Beck, A.T., Brown, G.B. y Steer, R.A. (1987). Taking the measure of anxiety and depression: validity of reconstructed Hamilton Scales. J. Nerv. Ment. Dis., 175, 474-479.

Sharp, D., Power, K., Simpson, R., Swanson, V., Moodie, E., Anstee, J. y Ashford, J. (1996). Fluvoxamine, pplaceb, and cognitive behavior therapy used alone and in combination in the treatment of panic disorder and agoraphobia. Journal of anxiety Disorders, 10, 219-242.

Spielberger, C.D., Gorsuch, R.L. y Lushene, R.E. (1970). Manual for the state-trait anxiety inventory. Palo Alto, California: Consulting Psychologists Press. (TEA, 1988).

Telch, M.J. (1988a). Combined pharmacological and psychological treatments. En C.G. 
Last y M. Hersen (Eds.), Handbook of anxiety disorders. New York: Pergamon. Telch, M.J. (1988b). Combined pharmacological and psychological treatments for panic sufferers. En S. Rachman y J.D. Maser (Eds.), Panic: Psychological perspectives. Hillsdale: Erlbaum.

Telch, M.J., Agras, W.S., Taylor, C.B., Roth,
W.T. y Gallen, C.C. (1985). Combined pharmacological and behavioral treatment of agoraphobia. Behavior Research and Therapy, 23, 325-335.

Zitrin, C.M. (1983). Differential treatment of phobias: Use of imipramine for panic attacks. Journal of Behavior Therapy and Experimental Psychiatry, 14, 11-18. 